\title{
Formation of magnetite in Magnetospirillum gryphiswaldense studied with FORC diagrams
}

\author{
Claire Carvallo ${ }^{1}$, Stanislawa Hickey $^{1}$, Damien Faivre ${ }^{2,3}$, and Nicolas Menguy ${ }^{1}$ \\ ${ }^{1}$ Institut de Minéralogie et de Physique des Milieux Condensés, Campus Boucicaut, 140 rue de Lourmel, 75015 Paris, France \\ ${ }^{2}$ Max Planck Institute for Marine Microbiology, Celsisusstr. 1, 28359 Bremen, Germany \\ ${ }^{3}$ Max Planck Institute of Colloids and Interfaces, Department of Biomaterials, Science Park Golm, 14424 Potsdam, Germany
}

(Received November 19, 2007; Revised March 11, 2008; Accepted March 20, 2008; Online published January 23, 2009)

\begin{abstract}
In order to study the formation of magnetite in magnetotactic bacteria, FORC diagrams were measured on a set of cultured Magnetospirillum gryphiswaldense, following an assay in which the iron uptake is used only for magnetite formation and not for cell growth. This enabled us to follow the magnetite formation independently of growth. The FORC diagrams showed a clear evolution from a size-distribution with a majority of superparamagnetic grains, to a distribution dominated by stable, single-domain grains, but still containing some superparamagnetic particles. TEM observations confirm this evolution. According to the saturation isothermal remanent magnetization cooling and warming curves, the Verwey transition can only be seen in the most mature samples, and slightly below $120 \mathrm{~K}$. This suggests that the samples may have suffered from some partial oxidation. Key words: Magnetotactic bacteria, FORC diagrams, magnetite.
\end{abstract}

\section{Introduction}

Magnetotactic bacteria (MTB) orient and migrate along the Earth magnetic field towards favorable habitats. Since the first report of MTB by Blakemore (1975), subsequent studies have shown that MTB are a phylogenetically and morphologically diverse group of aquatic microorganisms inhabiting freshwater and marine environments ranging from microaerobic to anoxic. Hallmarks of the MTBs are intracellular magnetosomes, which are magnetite $\left(\mathrm{Fe}_{3} \mathrm{O}_{4}\right)$ and occasionally greigite $\left(\mathrm{Fe}_{3} \mathrm{~S}_{4}\right)$ crystals enveloped in a membrane structure. Magnetosomes are characterized by narrow grain-size distributions (30-120 $\mathrm{nm}$ ), distinct species-specific crystal morphology, chemical purity and arrangement in single or multiple linear chains (Devouard et al., 1998; Bazylinski and Frankel, 2004; Thomas-Keprta et al., 2000; Faivre et al., 2008). Magnetic properties of magnetosomes are of great interest for a number of reasons. For example, in environmental magnetism, fossil magnetosomes can significantly contribute to the magnetic properties of some sediments and soils (Petersen et al., 1986; Chang and Kirschvink, 1989). They are also novel sources for fundamental studies in fine-grain magnetism and magnetic materials (Coker et al., 2007), and in biotechnologies (Lang et al., 2007; Matsunaga et al., 2007).

In the perspective of this special issue on the magnetism of volcanic rocks, one of the main current interests in biomagnetism resides in the possible biological origin of magnetite crystals on the ALH84001 Martian meteorite (McKay et al., 1996; Faivre and Zuddas, 2006). It was shown that the nanodimensional magnetite grains found in fracture

Copyright (c) The Society of Geomagnetism and Earth, Planetary and Space Sciences (SGEPSS); The Seismological Society of Japan; The Volcanological Society of Japan; The Geodetic Society of Japan; The Japanese Society for Planetary Sciences; TERRAPUB. zones of the meteorite are similar in size and shape to those in the magnetosomes of terrestrial magnetite bacteria. From transmission electron microscope analysis of single-crystals extracted from the ALH84001 Martian meteorite, ThomasKeprta et al. (2000) concluded that about $25 \%$ of the magnetite crystals in the zoned carbonate are likely magnetofossils mixed with $75 \%$ of inorganic magnetite. However, the observation of magnetosomes chains, which is one property unique to biogenic magnetite, is more difficult to put in evidence. While Friedmann et al. (2001) claimed to have identified magnetosome chains on scanning electron microscope images, Weiss et al. (2004) showed with magnetic measurements that only $10 \%$ of magnetite in ALH84001 could be isolated in chains. However there is still considerable debate about this matter (Kopp and Kirschvink, 2007).

In this paper, we study the formation of magnetite in Magnetospirillum gryphyswaldense, using first-order reversal curve (FORC) diagrams. FORC diagrams of SD particles can be interpreted as a combination of coercivity distributions (and therefore grain size distributions in the case of a single magnetic mineral) and interaction field distributions. Therefore, they are the ideal tool for this studying the evolution of magnetite crystals through time, as well as the chain formation. The samples were grown using an assay in which magnetite formation can be studied independently from cell growth.

\section{Samples and Preliminary Measurements}

The growth conditions are described in details in Faivre et al. (2007, 2008). Briefly, cells were first grown in a low iron medium, in which formation of magnetite was repressed. Then, cells were transferred to a medium in which further growth was stopped but formation of magnetite was enabled. At given time intervals, samples were withdrawn 

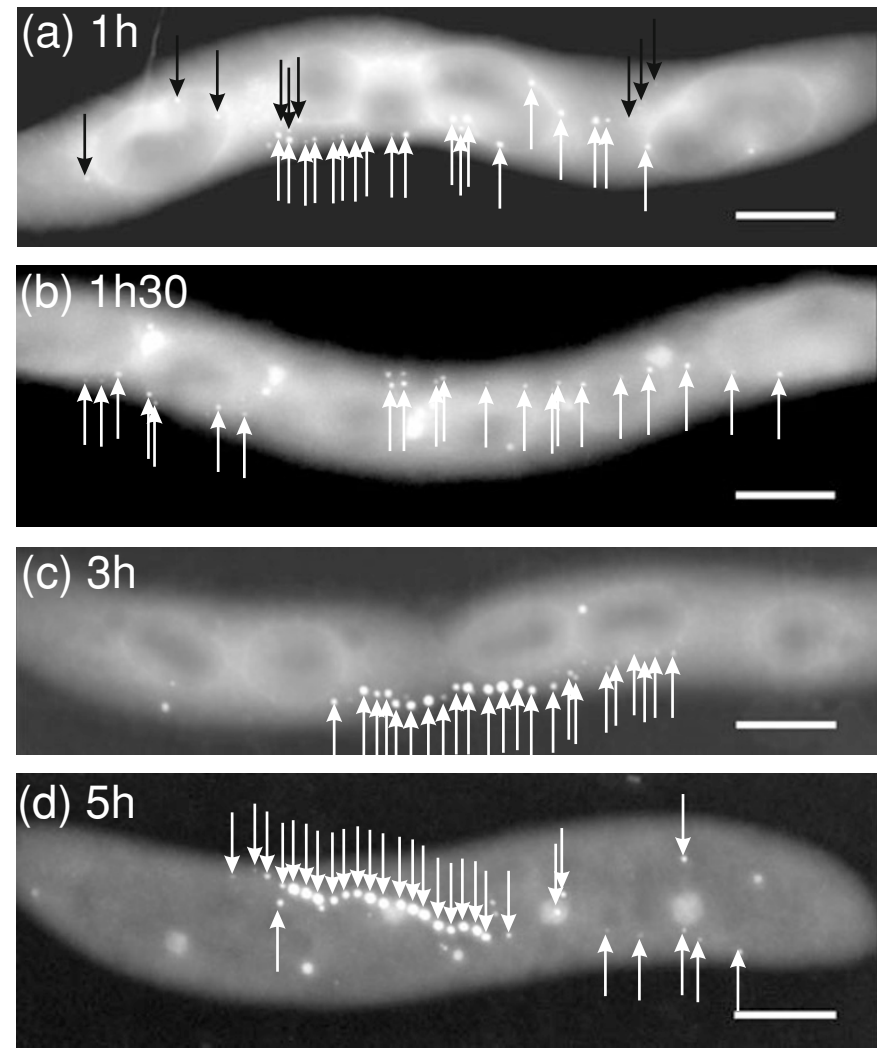

Fig. 1. TEM images of a time-course series of M. Gryphiswaldense. The scale bar is $500 \mathrm{~nm}$. Arrows point to what we have identified as magnetosomes.

Table 1. Average, minimum and maximum sizes for each of the timecourse samples. The values in brackets give the range of values.

\begin{tabular}{ccc}
\hline Growth time & Average size $(\min -\max )(\mathrm{nm})$ & Nearest-neighbour distance $(\min -\max )(\mathrm{nm})$ \\
\hline $1 \mathrm{~h}$ & $21.7(8.4-43.5)$ & $192.0(12.2-928.5)$ \\
$1.5 \mathrm{~h}$ & $25.1(12.4-39.7)$ & $181.0(19.2-801.7)$ \\
$3 \mathrm{~h}$ & $33.6(12.4-55.6)$ & $120.7(9.7-822.8)$ \\
$5 \mathrm{~h}$ & $39.8(12.8-64.8)$ & $55.1(5.7-546.0)$ \\
\hline
\end{tabular}

from the culture for measurements. For analysis, samples were then centrifuged and dried in order to obtain $5 \mathrm{mg}$ whole cells samples.

High Resolution Electron Microscope (HREM) observations were performed on a JEOL 2100F microscope operating at $200 \mathrm{kV}$ and equipped with a field emission gun, a high-resolution UHR pole piece, and a Gatan GIF 2001 energy filter. A drop containing the magnetotactic bacteria was deposited onto a carbon coated 200 mesh copper grid. The excess of water was removed with an absorbing paper and the remaining water was pumped in the airlock chamber of the microscope. An example of images through the course of the bacteria growing is given in Fig. 1. It shows an evolution from bacteria without any magnetite crystal to several chains of well-formed magnetite crystals. In this series, the first crystals started forming at $t=1 \mathrm{hr}$.

In order to obtain the distributions of sizes and distances between particles, we measured the grain diameter and the edge-edge distance of nearest-neighbors for about 150 grains in several different cells from the HREM photos, for each of the 4 different samples. According to the results given in Table 1 and Fig. 2, and as predicted, the grain size increases with time, and the nearest-neighbor distance de- creases with time. The most important change occurs between $t=1 \mathrm{~h} 30 \mathrm{mn}$ and $t=3 \mathrm{~h}$, which is the time period where the chains are suddenly almost completely formed. This is in agreement with what was observed by Scheffel $e t$ al. (2006) and Komeili et al. (2006). The presence of small grains remaining at the extremities of the chain in the most mature stage is consistent with electron holography observations (Dunin-Borkowski et al., 1998) and other TEM observations (Faivre et al., 2007)

We compared our results with results from the squareness-coercivity plot (Tauxe et al., 2002). The squareness is defined as $M_{\mathrm{rs}} / M_{\mathrm{s}}$. We prefer this plot to the traditional Day-plot because of the difficulty in estimating the coercivity of remanence. When compared with the modeled values of Tauxe et al. (2002), our values fall close to the cubic SD-SP mixing line (Fig. 3(a)). We also measured ARM/SIRM ratios. ARMs were given in a $100 \mu \mathrm{T}$ field with a $60 \mathrm{mT}$ alternating-field, and SIRMs were given in a $300 \mathrm{mT}$ field. The ARM/SIRM ratio clearly increases when the time in the growth medium increases (Fig. 3(b)), showing an increasing SD population with time in the growth medium. 

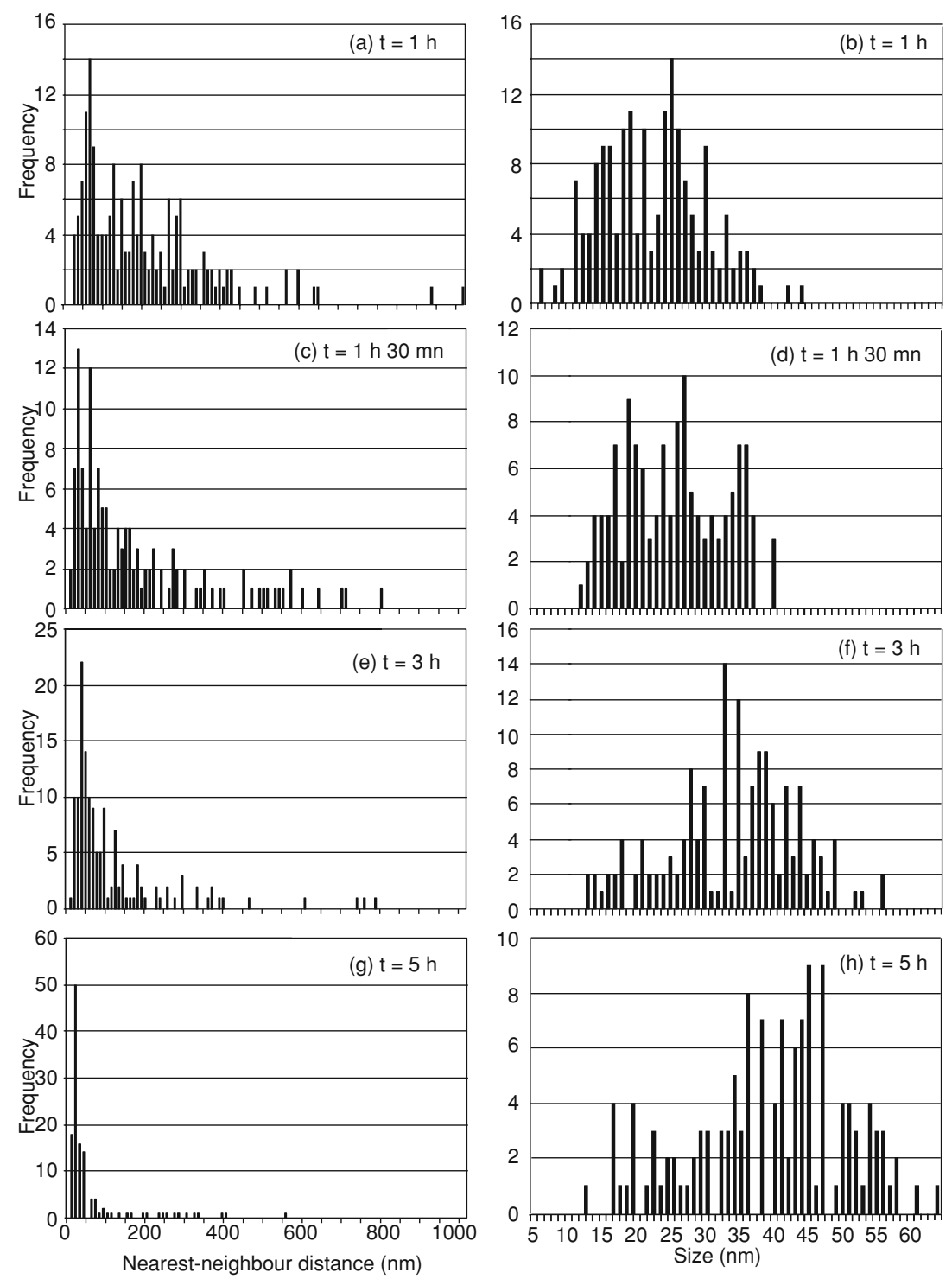

Fig. 2. Nearest-neighbor distances between the edges of the grains (left) and grain size (right) distributions for the five samples. These statistics refer to magnetosomes contained in about 10 cells.

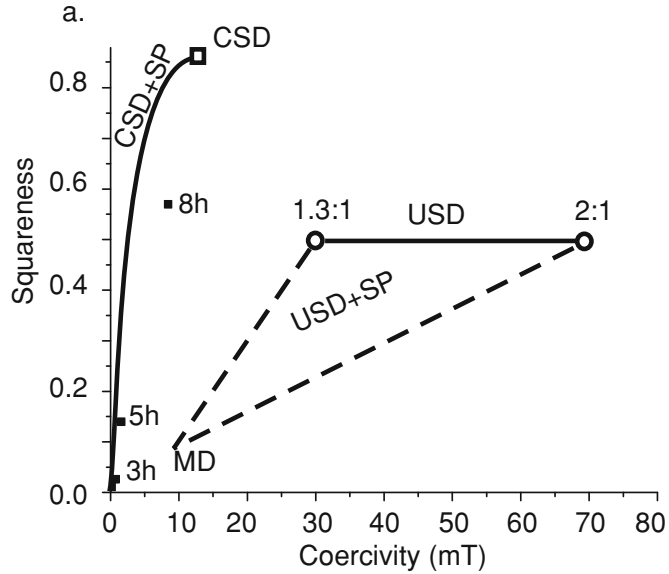

b.

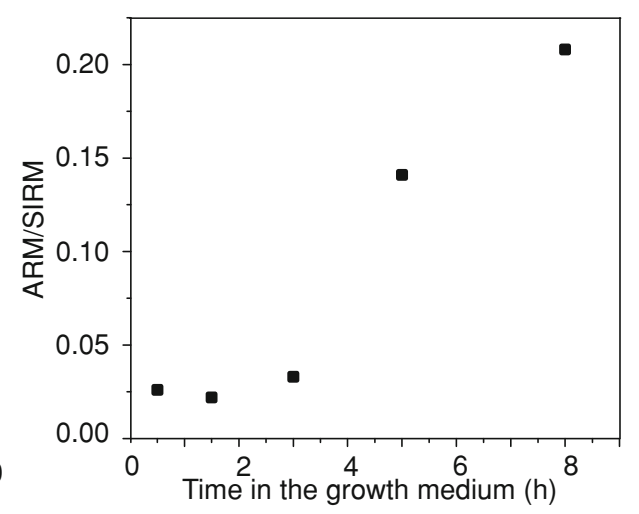

Fig. 3. Left: Squareness-coercivity plot of our samples, and comparison with the theoretical predictions of Tauxe et al. (2002) for randomly oriented populations of uniformly magnetized magnetite. Re-drawn from Tauxe et al. (2002). CSD: cubic single-domain; USD: uniaxial single-domain with length to width ratio of 1.3 and 2. Right: ARM/SIRM as a function of the time in the growth medium. SIRM was imparted in a $0.3 \mathrm{~T}$; ARM was imparted in a $100 \mu \mathrm{T}$ field with a $60 \mathrm{mT}$ alternating-field. 

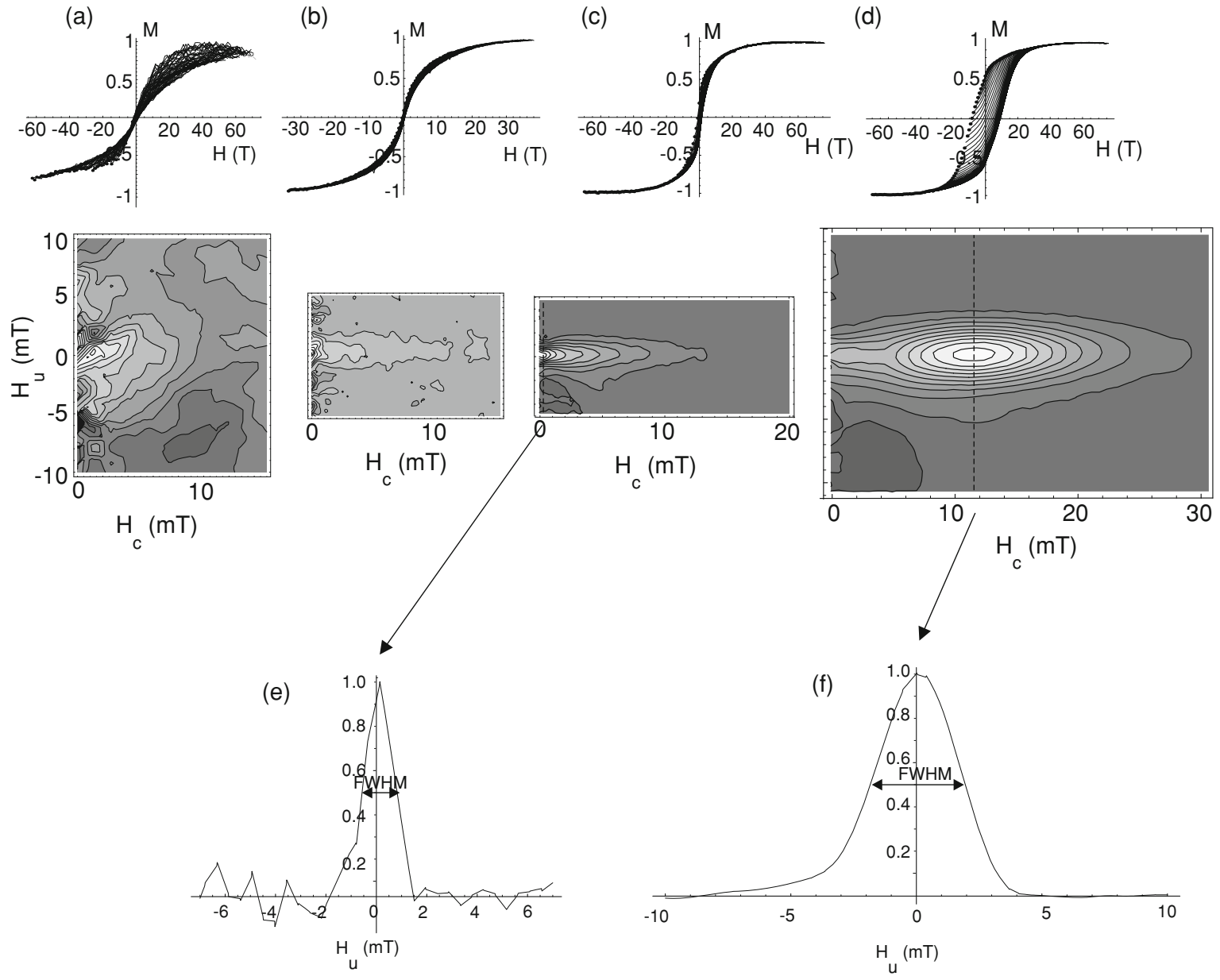

Fig. 4. FORCs (top) and FORC diagrams (bottom) of the time course series of $M$. gryphiswaldense after: (a) $1 \mathrm{~h} 30$; (b) 3 h; (c) 5 h; (d) 8 h in the growth medium. $\mathrm{SF}=4$ for all the FORC diagrams. FORC profiles through the maximum of coercivity (indicated by the dotted lines on the FORC diagrams) parallel to the vertical axis for the (e) $t=5 \mathrm{~h}$; (f) $t=8 \mathrm{~h}$ samples.

\section{FORC Diagrams}

FORC diagrams are constructed by measuring a large number of partial magnetic hysteresis curves called firstorder reversal curves (FORCs) (Pike et al., 1999; Roberts et al., 2000). Starting at positive saturation, the applied field is decreased until a specified reversal field $H_{\mathrm{r}}$ is reached. A FORC is the magnetization curve measured at regular field steps from $H_{\mathrm{r}}$ back to positive saturation. The FORC distribution is defined as the second mixed derivative:

$$
\rho\left(H_{\mathrm{a}}, H_{\mathrm{b}}\right)=-\frac{\partial^{2} M\left(H_{\mathrm{r}}, H\right)}{\partial H_{\mathrm{r}} \partial H},
$$

where $M\left(H_{\mathrm{r}}, H\right)$ is the magnetization measured at $H$ on the FORC with reversal field $H_{\mathrm{r}}$. The FORC distribution is then plotted on the coordinates $H_{\mathrm{c}}=\left(H_{\mathrm{b}}-H_{\mathrm{a}}\right) / 2$ and $H_{\mathrm{u}}=\left(H_{\mathrm{b}}+H_{\mathrm{a}}\right) / 2$. A more complete explanation of the measurement and construction of FORC diagrams is given by Muxworthy and Roberts (2006). The interpretative framework of FORC diagrams are detailed in Pike et al. (1999) and Roberts et al. (2000). It has been confirmed based on measurements on well characterized natural and synthetic samples as well as micromagnetic modeling (Carvallo et al., 2003; Muxworthy and Williams, 2005).

In the case of ideal SD grain assemblages, under the assumption that $H_{\mathrm{c}}$ and $H_{\mathrm{u}}$ are fixed for each grain but differ- ent from grain to grain, the FORC function is equivalent to a Preisach function (Preisach, 1935) and according to Néel's interpretation (Néel, 1954), the distribution along the $H_{\mathrm{c}}$ axis is equivalent to the distribution of particle microcoercivities, and a cross-section through the peak of the FORC distribution parallel to the $H_{\mathrm{i}}$ axis left of the central peak is equivalent to the distribution of magnetostatic interaction fields. More generally, the accurate representation of the coercivity distribution of SD particles is the marginal distribution (Egli, 2006; Winklhofer and Zimanyi, 2006). A recent study by Chen et al. (2007) on intact and disrupted chains of magnetite magnetosomes with various levels of dipolar inter-particle and inter-chain interactions efficiently predicted packing fraction and dipolar interaction distribution from FORC diagrams.

All these previous studies have showed the following patterns on FORC diagrams. SD particles are characterized by closed concentric contours about a central peak. If the magnetic interactions are strong, the contours show a much greater spread parallel to the $H_{\mathrm{i}}$ axis than those with less interactions. FORC diagrams for multi-domain (MD) particles go from contours that diverge away from the horizontal axis close to the origin, to nearly vertical contours with the peak close to $H_{\mathrm{c}}=0$. Finally, fine-grained SD particles are not blocked at room temperature and undergo vary- 
ing degrees of thermal relaxation depending on their volume. This translates on the FORC diagram into the shift of the SD FORC distribution to lower coercivities, so that the FORC distribution intersects the $H_{\mathrm{i}}$ axis. The manifestation of these superparamagnetic (SP) particles on the FORC diagram is due to the fact that the measurement time is faster than the relaxation time.

FORC diagrams were measured at room-temperature on a Princeton Alternating Gradient Magnetometer (AGM) at the Institute for Rock Magnetism (University of Minnesota) and at the Laboratoire des Sciences du Climat et de l'Environnement (Gif-sur-Yvette, France). One hundred FORCs were measured to construct each FORC diagram, and the averaging time was set between 0.1 and $0.5 \mathrm{~s}$, depending on the noise level. The smoothing factor (SF) was set to 4 for all the FORC diagrams. The FORC measurements were carried out on a series different from the series used for TEM imaging. The modes of preparation were similar but the time scales are likely to be different. The cells were centrifuged at $5000 \mathrm{rpm}$, then the supernatant was removed and the remaining was left to dry for a day. The time between growth and measurement was about two weeks. The totality of the dried sample was then deposited on the AGM probe for measurement.

In the case of chains of magnetite particles, once individual particles are less than three particle diameters away from its nearest neighbor in any given chain, FORC diagrams can only be interpreted on the basis of coercivity and dipolar magnetostatic interaction distribution among chains and/or among chains and individual particles not in chains. As individual particles behave collectively during hysteresis measurements due to the strong coupling of positive interparticle dipolar magnetostatic interactions, FORC diagrams can not be interpreted on the basis of individual particles in magnetosome chains (Penninga et al., 1995; Pan et al., 2005; Chen et al., 2007; Prozorov et al., 2007).

Because of a too weak magnetization, it was impossible to measure FORC diagrams or even a hysteresis loop for the $t=0$ and $t=30 \mathrm{mn}$ samples. The FORC diagram at $t=2 \mathrm{~h}$ (Fig. 4(a)) is extremely noisy, even though it was measured with an averaging time of $0.5 \mathrm{~s}$ at each point. We can distinguish a peak close to the $H_{\mathrm{c}}=0$ axis, which corresponds to a SP contribution. This is confirmed by the data from the hysteresis loop: the ratio of saturation remanent magnetization over the saturation magnetization is 0.015 , and the bulk coercivity is very close to zero. At $t=$ $3 \mathrm{~h}$ (Fig. 4(b)), the FORC diagram is much less noisy, and the interpretation is more straightforward: the peak of the distribution is still close to the $H_{\mathrm{c}}=0$ axis, but a tail starts to appear, which corresponds to a small contribution from a stable SD component. This pattern continues its evolution in the same direction, as the high coercivity tail becomes more important on the $t=5 \mathrm{~h}$ FORC diagram (Fig. 4(c)). Finally, at $t=8 \mathrm{~h}$ (Fig. 4(d)), the main pattern is a clear, single peak characteristic of SD grains. However, the lowest contours are not all closed, but they intersect the $H_{\mathrm{c}}=0$ axis, indicating that a SP contribution remains. At $t=5$ and $8 \mathrm{~h}$, a negative peak is present close to the $H_{\mathrm{c}}=0$ axis, in the negative $H_{\mathrm{i}}$ region, which is different from noise. This negative feature provides another evidence for the presence of SD grains (Carvallo et al., 2004; Newell, 2005). It is caused by the decrease of $\partial M / \partial H_{\mathrm{r}}$ with decreasing $H$, resulting in a positive mixed derivative value and therefore a negative FORC distribution (Newell, 2005).

Between $t=5$ and $t=8 \mathrm{~h}$, the spread of the FORC distribution through the peak of maximum distribution, parallel to the $H_{\mathrm{i}}$ axis, increases. The full-width at halfmaximum goes from 2.0 to $3.4 \mathrm{mT}$, indicating that interactions between chains or between the chains and isolated grains (some of them constituting probably the SP component) also increase with time (Fig. 4(e), (f)).

\section{Low-temperature Measurements}

Cooling and warming curves of saturation isothermal remanent magnetization (SIRM) are used to identify lowtemperature transitions. In magnetotactic bacteria, the Verwey transition often occurs at a temperature lower than in stoechiometric magnetite (e.g., Moskowitz et al., 1993), and depends on the magnetosome morphology and blocking volume (Prozorov et al., 2007). In order to identify a possible Verwey transition and to measure $T_{\mathrm{V}}$, we carried out low-temperature measurements using a Magnetic Properties Measurement System at the Institute for Rock Magnetism, on the exact same samples as those used for the FORC diagram measurements. We collected measurements of temperature dependent SIRM given with a $2.5 \mathrm{~T}$ applied magnetic field at $300 \mathrm{~K}$. The $300 \mathrm{~K}$ SIRM were monitored both during cooling from $300 \mathrm{~K}$ to $10 \mathrm{~K}$ and during warming from $10 \mathrm{~K}$ to $300 \mathrm{~K}$. For the samples corresponding to $t<5 \mathrm{~h}$, the noise is too important to obtain a meaningful measurement. The two most mature samples $(t=5$ and $t=$ $8 \mathrm{~h}$ ) show markedly different behaviours. In the first case, the SIRM cooling and warming curve is very similar to that observed by Pan et al. (2005) for uncultured nagnetotactic bacteria containing a large proportion of Magnetobacterium bavaricum (Fig. 5(a)). First, the remanence increases up to about $130 \mathrm{~K}$, then decreases slightly when the temperature is decreased down to $10 \mathrm{~K}$. Upon warming, the remanence is reversible up to about $130 \mathrm{~K}$, then decreases when the temperature is increased up to $300 \mathrm{~K}$, but the remanence at the end of the cycle is about $2 \%$ lower than the initial SIRM $_{300 \mathrm{~K}}$. The shape of the SIRM cooling-warming curve for the sample at $t=8 \mathrm{~h}$ is much more consistent with what is expected for SD magnetite (Özdemir et al., 2002), though on a much smaller scale (Fig. 4(b)). However, it has to be noted that the lost in remanence in the case of Özdemir et al. (2002) could have been cause by grains larger than SD grains. There is a large drop in magnetization upon cooling through the Verwey transition, then a slight increase. This behavior is reversible up until $50 \mathrm{~K}$, then the remanence drops slightly lower than on the cooling curve, and a part of the remanence is not recovered when the temperature is back to $300 \mathrm{~K}$ : the loss of remanence after the lowtemperature cycling is about $13 \%$.

The Verwey temperatures $\left(T_{\mathrm{V}}\right)$ were estimated by finding the temperature at which $d M / d T$ is maximum on the SIRM warming curve. The curve for $t=5 \mathrm{~h}$ was too noisy to yield a reliable $T_{\mathrm{V}}$ estimate. However, at $t=8 \mathrm{~h}, T_{\mathrm{V}}$ could be estimated at $115 \mathrm{~K}$ from the minimum of the $d M / d T$ curve. 
(a)

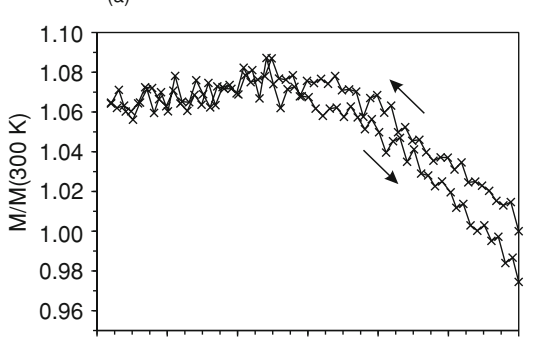

(c)

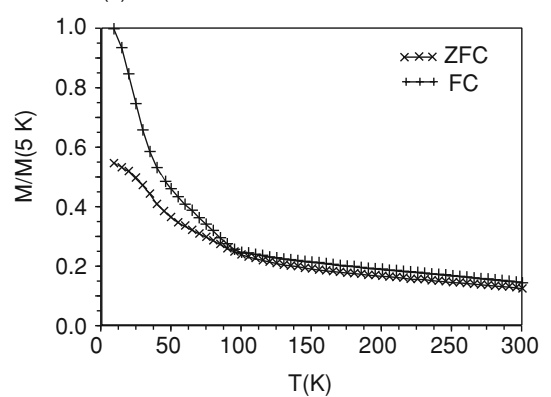

(b)

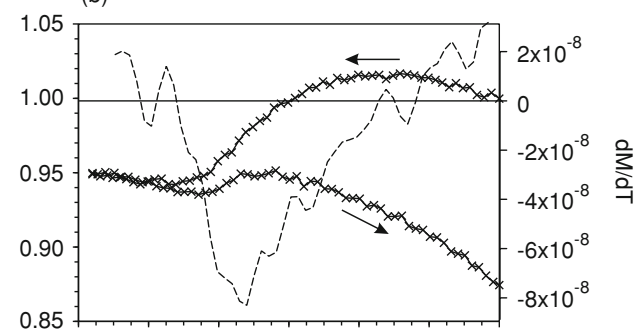

(d)

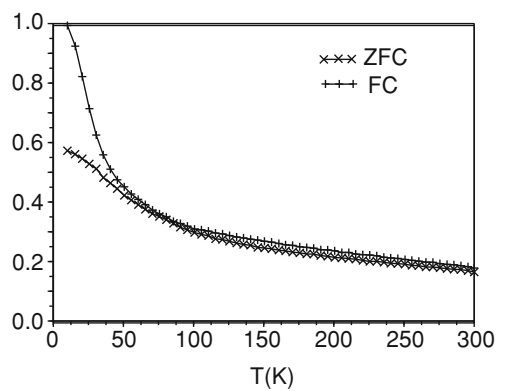

Fig. 5. Top: SIRM cooling and warming curves of $M$. gryphiswaldense after: (a) $5 \mathrm{~h}$; (b) $8 \mathrm{~h}$ in the growth medium; Bottom: FC/ZFC curves after (c) $5 \mathrm{~h}$; (d) $8 \mathrm{~h}$ in the growth medium. $d M / d T$ that was used to calculate $T_{\mathrm{V}}$ is also plotted on (b).

Warming curves of SIRM in zero-field after cooling both in zero-field (ZFC) and in a $2.5 \mathrm{~T}$ field (FC) were measured on the MPMS (Fig. 5(c), (d)). This measurement of SIRM induced at $10 \mathrm{~K}$ monitored on warming following two types of cooling pre-treatment is different from the previous measurement, which was that of the SIRM given at $300 \mathrm{~K}$ monitored on cooling and warming. The Verwey transition is absent from these two curves.

\section{Discussion}

By inducing magnetite nucleation and growth in resting, iron-starved cells of Magnetospirillum gryphiswaldense, we were able to follow the magnetosome development by FORC diagram, TEM and low-temperature measurements. Because the amount of magnetic material was very weak in the initial stages of the development, the magnetic measurements could only be performed for the final stages of growth. The Verwey temperature could only be calculated in one case and leads a value slightly lower than $120 \mathrm{~K}$. Nevertheless, the grain size evolution, from mainly SP to mainly SD, is very clear on the FORC diagrams, and is consistent with the observations from MET images.

It must be noted that the MET images and the FORC diagrams have been measured on two different series. Even though the culture method was identical, there could have been some differences in the cultures, which would explain that the two series did not develop at the same speed. For example, the series used for MET imaging seem to have grown faster than the series used for FORC diagram measurements: at $t=5 \mathrm{~h}$, according to the grain sizes measured on the MET images, the majority of the grains should be stable SD, but on the FORC diagram, the main contribution at the same time comes from SP particles.

Even though the TEM and the FORC samples are not the same, we can try to compare the interaction field given by the FORC diagram and that calculated from the nearest- neighbor distances measured from the TEM images for the most mature sample. The dipole field equation gives

$$
H_{\mathrm{int}} \approx \frac{\mu}{4 \pi r^{3}}
$$

where $r$ is the centre-to-centre separation (Dunlop and Özdemir, 1997). Using the average grain size of $39.8 \mathrm{~nm}$ and the centre-to-centre distance between nearestneighbours of $94.9 \mathrm{~nm}$, we find that the interaction field is about $2 \mathrm{mT}$. This is in the same order of magnitude as the FWHM (3.4 mT), though lower. However, since the particles are on average less than 3 particle diameter away from each other, we can only interpret the FWHM data as the interaction between chains and between chains and individual particles. In any case, the amount of interactions, from this crude calculation or from the FWHM, is quite small. Moreover, the shape of the FORC distribution for the most mature sample resembles the modelled FORC distribution of noninteracting, thermally activated SD grains (Egli, 2006).

The absence of Verwey transition at $t=5 \mathrm{~h}$ and the fact that it is at a temperature lower than $120 \mathrm{~K}$ at $t=8 \mathrm{~h}$ could be due to several factors. First, it could be due to the fact that, according to the FORC diagram, some grains are still SP at room temperature. If not enough grains are blocked around $120 \mathrm{~K}$, the Verwey transition will not be seen, which could be the case here. This is similar to what Moskowitz et al. (1989) observed on SP magnetite formed by dissimilatory iron-reducing bacteria. It could also be an effect of partial oxidation (Özdemir et al., 1993). Despite the fact that the magnetosomes can protect to some extent the magnetite crystals from being oxidized, some maghemitization can occur (Pan et al., 2005; Kasama et al., 2006; Prozorov et al., 2007). Even though a complete transformation from magnetite to maghemite would take years (Tang et al., 2003) and is not possible in our samples, according to Özdemir et al. (1993), "minor surface oxidation suppresses 
the Verwey transition".

The coercivity peak of the SD fraction is around $12 \mathrm{mT}$ (Fig. 4(d)). This is much lower than the typical coercivity known for magnetosomes, often over $40 \mathrm{mT}$ (e.g., Moskowitz et al., 1993; Chen et al., 2007). This high coercivity is often used as a hallmark of biogenic magnetite in coercivity component analysis (e.g., Egli, 2003). The particular laboratory conditions in which the magnetite is formed in this study might be at the origin of the discrepancy, but in any case we have shown that bacterial SD magnetite can have very low coercivities.

Altogether, our results suggest the following evolution: the first grains of magnetite formed are very small (in the SP range) and far away from each other. As more iron is added to the culture, magnetic grain size increases and more and more grains become SD, and start forming chains. When most grains are large enough to be SD, and arranged in chains, there still remains some SP grains. According to the TEM images, some of them are at the extremities of the chains, but the SP contribution on the FORC diagrams indicates that there should also remain some SP grains far enough from the chains so that they behave independently from the SD chains (Faivre et al., 2007, 2008).

Acknowledgments. We thank Carlo Laj and Catherine Kissel from the Laboratoire des Sciences du Climat et de l'Environnement (LSCE, Gif-sur-Yvette), for access to the AGM. Most of the FORC diagrams were measured at the Institute for Rock Magnetism, which is supported by the University of Minnesota, the Keck Fundation and the NSF Earth Sciences Division. We thank Mike Jackson, Pete Solheid and Brian Carter-Stiglitz for their help with the measurements. D. Faivre was supported by a Marie Curie Fellowship from the European Union (project Bac-Mag, EIF-2005-009637), and acknowledges current funding from the Max Planck Society. This paper has been greatly improved thanks to constructive comments from Amy Chen and an anonymous reviewer.

\section{References}

Bazylinski, D. A. and R. B. Frankel, Magnetosome formation in Prokaryotes, Nature Rev. Microbiol., 2, 217-230, 2004.

Blakemore, R. P., Magnetotactic bacteria, Science, 190, 377-379, 1975.

Carvallo, C., A. R. Muxworthy, D. J. Dunlop, and W. Williams, Micromagnetic modeling of first-order reversal curve (FORC) diagrams for single-domain and pseudo-single-domain magnetite, Earth Planet. Sci. Lett., 213, 375-390, 2003.

Carvallo, C., Ö. Özdemir, and D. J. Dunlop, First-order reversal curve (FORC) diagrams of elongated single-domain grains at high and low temperatures, J. Geophys. Res., 109, doi/ 10.1029/2003JB002539, 2004.

Chang, S. B. R. and J. L. Kirschvink, Magnetofossils, the magnetization of sediments and the evolution of magnetite biomineralization, Ann. Rev. Earth Planet. Sci., 17, 169-195, 1989.

Chen, A. P., R. Egli, and B. M. Moskowitz, First-order reversal curve (FORC) diagrams of natural and cultured biogenic magnetic particles, J. Geophys. Res., 112, doi/ 10.1029/2006JB004575, 2007.

Coker, V. S., C. I. Pearce, C. Lang, G. van der Laan, R. A. D. Pattrick, N. D. Telling, D. Schüler, E. Arenholz, and J. R. Lloyd, Cation site occupancy of biogenic magnetite compared to polygenic ferrite spinels determined by X-ray magnetic circular dichroism, Eur. J. Mineral., 19, 707-716, 2007.

Devouard, B., M. Posfai, X. Hua, D. A. Bazylinski, R. B. Frankel, and P. R. Buseck, Magnetite from magnetotactic bacteria: Size distributions and twinning, Am. Mineral., 83, 1387-1398, 1998.

Dunin-Borkowski, R. E., M. R. McCartney, R. B. Frankel, D. A. Bazylinski, M. Pósfai, and P. R. Buseck, Magnetic microstructure of magnetotactic bacteria by electron holography, Science, 282, 1868-1870, 1998.

Dunlop, D. and Ö. Özdemir, Rock Magnetism: Fundamentals and Frontiers. Cambridge Studies in Magnetism, Cambridge Univ. Press, 3, New
York, 1997.

Egli, R., Analysis of the field dependence of remanent magnetization curves, J. Geophys. Res., 108(B2), 2081, doi:10.1029/2002JB002023, 2003.

Egli, R., Theoretical aspects of dipolar interactions and their appearance in first-order reversal curves of thermally activated single-domain particles, J. Geophys. Res., 111, B12S17, doi:10.129/2001JB000671, 2006.

Faivre, D. and P. Zuddas, An integrated approach for determining the origin of magnetite nanoparticles, Earth Planet. Sci. Lett., 243, 53-60, 2006.

Faivre, D., L. H. Böttger, B. F. Matzanke, and D. Schüler, Intracellular Magnetite Biomineralization in Bacteria Proceeds by a Disctinct Pathway Involving Memberane-bound Ferritin and Iron-II Species, Angew. Chem. Int. Ed., 46, 8495-8499, doi:10.1002/anie.200700927, 2007.

Faivre, D., N. Menguy, M. Pósfai, and D. Schüler, Fast-growing magnetosomes exhibit lack of biological control over magnetite biomineralization, Am. Mineral., 93, 463-469, 2008.

Friedmann, E. I., J. Wierzchos, C. Ascaso, and M. Winklhofer, Chains of magnetite crystals in the meteorite ALH84001: Evidence of biological origin, Proc. Natl. Acad. Sci. USA, 98, 2176-2181, 2001.

Kasama, T., M. Pósfai, R. K. K. Chong, A. P. Finlayson, P. R. Buseck, R. B. Frankel, and R. E. Dunin-Borkowski, Magnetic properties, microstructure, composition and morphology of greigite nanocrystals in magnetotactic bacteria from electron holography and tomography, Am. Mineral., 91, 1216-1229, 2006.

Komeili, A., Z. Li, D. K. Newman, and G. J. Jensen, Magnetosomes are cell membrane invaginations organized by the actin-like protein MamK, Science, 311, 242-245, 2006.

Kopp, R. E. and J. L. Kirschvink, The identification and biogeochemical interpretation of fossil magnetotactic bacteria, Earth Sci. Rev., doi: 10.1016/j.earscirev.2007.08.001, 2007.

Lang, C., D. Schüler, and D. Faivre, Synthesis of Magnetite Nanoparticles for Bio- and Nanotechnology: Genetic Engineering and Biomimetics of Bacterial Magnetosomes, Macromol. Biosci., 7, 144-151, 2007.

Matsunaga, T., T. Suzuki, M. Tanaka, and A. Arakaki, Molecular analysis of magnetotactic bacteria and development of functional bacterial magnetic particles for nano-biotechnology, Trends Biotechnol., 25, 182-188, 2007.

McKay, D. S., E. K. Gibson Jr., H. Vali, C. S. Romanek, S. J. Clemett, X. D. F. Cilier, C. R. Maechling, and R. N. Zare, Search for Past Life on Mars: Possible Relic Biogenic in Martian Meteorite ALH84001, Science, 273, 924-930, 1996.

Moskowitz, B. M., R. B. Frankel, D. A. Bazylinski, H. W. Jannasch, and D. R. Lovley, A comparison of magnetite particles produced anaerobically by magnetotactic and dissimilatory iron-reducing bacteria, Geophys. Res. Lett., 16, 665-668, 1989.

Moskowitz, B. M., R. B. Frankel, and D. A. Bazylinski, Rock magnetic criteria for the detection of biogenic magnetite, Earth Planet. Sci. Lett., 120, 283-300, 1993.

Muxworthy, A. R. and W. Williams, Magnetostatic interaction fields in first-order reversal curve (FORC) diagrams, J. Appl. Phys., 97, 063905, 2005.

Muxworthy, A. R. and A. P. Roberts, First-order reversal curve (FORC) diagrams, in Encyclopedia of Geomagnetism and Paleomagnetism, edited by D. Gubbins and E. Herrero-Bervera, Springer, New York, 2006.

Néel, L., Remarques sur la théorie des propriétés magnétiques des substances dures, Appl. Sci.Res., B4, 13-24, 1954.

Newell, A. J., A high-precision model of first-order reversal curve (FORC) functions for single-domain ferromagnets with uniaxial anisotropy, Geochem. Geophys. Geosyst., 6, Q05010, doi: 10.1029/2004GC000877, 2005.

Özdemir, Ö., D. J. Dunlop, and B. M. Moskowitz, The effect of oxidation on the Verwey transition in magnetite, Geophys. Res. Lett., 20, 1671$1674,1993$.

Özdemir, Ö., D. J. Dunlop, and B. M. Moskowitz, Changes in coercivity, remanence, and domain state at low temperature in magnetite, Earth Planet. Sci. Lett., 194, 343-358, 2002.

Pan, Y., N. Petersen, M. Winklhofer, A. F. Davila, Q. Liu, T. Frederichs, M. Hanzlik, and R. Zhu, Rock magnetic properties of uncultured magnetotactic bacteria, Earth Planet. Sci. Lett., 237, 311-325, 2005.

Penninga, I., H. D. Waard, B. M. Moskowitz, D. A. Bazylinski, R. B. Frankel, and R. B. Frankel, Remanence measurements on individual magnetotactic bacteria using a pulsed magnetic field, J. Magn. Magn. Mater., 149, 279-286, 1995.

Petersen, N., T. van Dobeneck, and H. Vali, Fossil bacterial magnetite in deep sea sediments from the South Atlantic Ocean, Nature, 320(6063), 
611-615, 1986.

Pike, C. R., A. P. Roberts, and K. L. Verosub, Characterizing interactions in fine magnetic particle systems using first order reversal curves, J. Appl. Phys., 85, 6660-6667, 1999.

Preisach, F., Uber die magnetische Nachwirkung, Z. Phys., 94, 277-302, 1935.

Prozorov, R., T. Prozorov, S. K. Mallapragada, B. Narasimhan, T. J. Williams, and D. A. Bazylinski, Magnetic irreversibility and the Verwey transition in nanocrystalline bacterial magnetite, Phys. Rev. B, 76, 054406, 2007.

Roberts, A. P., C. R. Pike, and K. L. Verosub, FORC diagrams: a new tool for characterizing the magnetic properties of natural samples, $J$. Geophys. Res., 100, 17909-17924, 2000.

Scheffel, A., M. Gruska, D. Faivre, A. Linaroudis, J. M. Plitzko, and D. Schuler, An acidic protein aligns magnetosomes along a filamentous structure in magnetotactic bacteria, Nature, 440, 110-114, 2006.

Tang, J. T., M. Myers, K. A. Bosnick, and L. E. Brus, Magnetite $\mathrm{Fe}_{3} \mathrm{O}_{4}$ nanocrystals: Spectroscopic observation of aqueous oxidation kinetics, J. Phys. Chem. B, 107, 7501-7506, 2003.
Tauxe, L., H. Neal Bertram, and C. Seberino, Physical interpretation of hysteresis loops: Micromagnetic modeling of fine particle magnetism, Geochem. Geophys. Geosyst., 3, doi: 10.1029/2001GC000241, 2002.

Thomas-Keprta, K. L., D. A. Bazylinski, J. L. Kirschvink, S. J. Clemett, D. S. McKay, S. J. Wentworth, H. Vali, E. K. Gibson Jr., and C. S. Romanek, Elongated prismatic crystals in AL84001 carbonate globules: Potential Martian Magnetofossils, Geochim. Cosmochim. Acta, 64 4049-4081, 2000.

Weiss, B. P., S. S. Kim, J. L. Kirschvink, R. E. Kopp, M. Sankaran, A. Kobayashi, and A. Komeili, Magnetic tests for magnetosome chains in Martian meteorite ALH84001, Geochim. Cosmochim. Acta, 101, 82818284, 2004.

Winklhofer, M. and G. T. Zimanyi, Extracting the intrinsic switching field distribution in perpendicular media: A comparative analysis, J. Appl. Phys., 99, 08E710, doi:10.1063/1.2176598, 2006.

C. Carvallo (e-mail: carvallo@impmc.jussieu.fr), S. Hickey, D. Faivre, and N. Menguy 